\title{
Angelica polymorpha Maxim Induces Apoptosis of Human SH-SY5Y Neuroblastoma Cells by Regulating an Intrinsic Caspase Pathway
}

\author{
Md. Ataur Rahman, Kausik Bishayee, and Sung-Oh Huh*
}

\begin{abstract}
Angelica polymorpha Maxim root extract (APRE) is a popular herbal medicine used for treating stomachache, abdominal pain, stomach ulcers, and rheumatism; however the effect of APRE on cancer cells has not yet been explored. Here, we examined APRE cytotoxicity seen on target neuroblastoma cells (NB) using cell viability assays, DAPI visualization of fragmented DNA, and Western blotting analysis of candidate signaling pathways involved in proliferation and apoptosis. We demonstrated that APRE reduced cell viability in NB to a greater extent than in fibroblast cells. In addition, we found that APRE could inhibit the three classes of MAPK proteins and could also down-regulate the PISK/AKT/GSK-3ß activity all being relevant for proliferation and survival. APRE could also up-regulate Bax expression and down-regulate Bcl-2 and Mcl-1. With APRE treatment, depolarization of mitochondria membrane potential and activation of caspase-3 was demonstrated in the SH-SY5Y cells. We could not found increased activity of death receptor and caspase-8 as markers of the extrinsic apoptosis pathway for the APRE treated cells. In presence of a caspase-3 siRNA and a pan-caspase inhibitor, APRE could not reduce the viability of NB cells to a significant degree. So we predicted that with APRE, the intrinsic pathway was solely responsible for inducing apoptosis as we also showed that the non-caspase autophagy pathway or ER stress-ROS mediated pathways were not involved. These findings demonstrate that an intrinsic mitochondria-mediated apoptosis pathway mediates the apoptotic effects of APRE on SHSY5Y cells, and that APRE shows promise as a novel agent for neuroblastoma therapy.
\end{abstract}

\section{INTRODUCTION}

Neuroblastoma (NB) is a neoplasm of neuroendocrine origin

Department of Pharmacology, College of Medicine, Institute of Natural Medicine, Hallym University, Chuncheon 200-702, Korea

*Correspondence: s0huh@hallym.ac.kr

Received 31 August, 2015; revised 21 October, 2015; accepted 26 October, 2015; published online 15 December, 2015

Keywords: Angelica polymorpha, apoptosis, Bax/Bcl-2 ratio, caspase, neuroblastoma and is aggressive in nature at the late stages of malignancy (high risk groups) (Pinto et al. 2015). The high-risk disease is difficult to treat even with the most aggressive multi-prolonged therapies available (Brodeur, 2003; Maris et al., 2008; Schrey et al., 2015), and there is a need for novel compounds to improve patient survival. Currently, diverse therapeutic approaches have been adopted to stop or slow the tumorigenic process by directly inhibiting proliferation of tumor cells, blocking angiogenesis, reducing metastasis, and by inducing tumor cell differentiation. Among them the tumor differentiating therapies that depend on the Erk MAPK have shown some promise on high-risk NB such as 13-cis-retinoic acid (Sonawane et al., 2014) and is now undergoing in phase 1 clinical trial (for detail see NCT01208454 at http://www.clinicaltrials.gov). In addition, we found an Erk-MEK inhibitor gambogic acid [approved by Chinese Food and Drug Administration for phase II clinical trial in solid cancer therapy (Shi et al., 2014)] that was effective against NB cells (Rahman, 2013). Here after extensive screening, we found the extract of Angelica polymorpha Maxim could inhibit NB cells by inducing apoptosis and showed multi-MAPK inhibitory effect.

Angelica polymorpha Maxim, the plant of Umbelliferae, is distributed throughout the North Temperate Zone (China, Korea, Republic of Korea, Japan), and New Zealand. In traditional medicine, the root of $A$. polymorpha can be used alone for treating chronic gastritis, stomach ache, abdominal pain, rheumatism aches, gastric ulcers and duodenal bulbar ulcers (Wang et al., 2009). The active components of Angelica polymorpha Maxim include coumarins, terpenoids, and volatile oils such as imperatorin, isoimperatorin oxypeucedanin, psoralen, oxypeucedanin hydrate, byakangelicin, angeliticin, $\alpha$-pinene, myrcene, and p-cymene (Yang et al., 2013). The crude extracts of Angelica polymorpha have shown strong anti-ulcer activity in vivo, and further separation of the crude extracts led to the isolation of bisabolangelone (Wang et al., 2009). However, there is no literature reporting of this plant for its anticancer activity.

Though APRE may be rich with various unknown anticancer substances, the precise molecular mechanism(s) of APRE against aggressive human NB cells has not yet been elucidated. Here we present results aimed at testing APRE effect on proliferative potential of NB cells via affecting different MAPKs at the protein level and the molecular mechanisms affecting proliferation. We found that APRE could elevate the expression of proapoptotic proteins Bax and caspases in induction of apoptosis in the SH-SY5Y-NB cells. Moreover, we showed that APRE showed specificity towards SH-SY5Y cancer cells and not 
NIH3T3 non-cancer fibroblast cells, and this property of APRE may make this drug more specific for cancer therapy.

\section{MATERIALS AND METHODS}

\section{Materials and cell culture}

Roots of Angelica polymorpha were purchased from DeaGuang in Chuncheon, South Korea. A voucher specimen (HRIC-1034) was deposited at the Regional Innovation Center, Hallym University, Chuncheon, South Korea. Roots of $A$. polymorpha $(1,000 \mathrm{~g})$ were chopped and blended using a Waring blender and then boiled with $2 \mathrm{~L}$ of $80 \%$ ethanol at $80^{\circ} \mathrm{C}$ for $2 \mathrm{~h}$. The insoluble materials were removed through centrifugation at $10,000 \times \mathrm{g}$ for $30 \mathrm{~min}$, and the resulting supernatant was concentrated and freeze-dried to yield a dark brown residue (Yield: $23.5 \%$ ). The freeze-dried residue was dissolved in dimethyl sulfoxide (DMSO) at a stock concentration of $10 \mathrm{mg} / \mathrm{ml}$ and subsequently diluted in medium to obtain the working concentration. Dulbecco's Modified Eagle's Medium (DMEM) and fetal bovine serum (FBS) were obtained from Gibco/BRL (USA). Antibodies against Fas, FasL and Mcl-1 were obtained from Santa Cruz Biotechnology (USA). Cleaved caspase-3, caspase-8, Bax, Bcl-2, $\beta$-actin, phospho-GSK-3 $\alpha$, phospho-GSK-

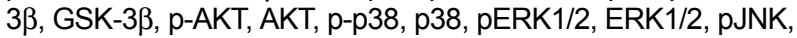
JNK, IRE1 $\alpha$, Ero1 $\alpha$, BiP, PERK and LC3 were obtained from Cell Signaling Technology (USA). DEVD-fmk was obtained from BD Biosciences. All other reagents were of analytical grade or of the highest purity available.

Human SH-SY5Y neuroblastoma, rat B103 neuroblastoma, Rat-2 fibroblast and NIH 3T3 mouse embryonic fibroblast cells were grown at $37^{\circ} \mathrm{C}$ in a humidified atmosphere of $5 \% \mathrm{CO}_{2}$. The cells were cultured in Dulbecco's Modified Eagle's Medium (DMEM) supplemented with $10 \%$ fetal bovine serum, $50 \mathrm{U} / \mathrm{ml}$ penicillin and $50 \mu \mathrm{g} / \mathrm{ml}$ streptomycin.

\section{Cell viability assay}

Cell viability was determined using a cytotoxicity assay kit, CCK-8 (Dojindo Molecular Technologies, Japan) according to the manufacturer's protocol. The cells were plated into 96 -well plates at a density resulting in $50-60 \%$ confluence and then treated with various concentrations of APRE. After treatment for $24 \mathrm{~h}, \mathrm{CCK}-8(10 \mu \mathrm{l})$ was added to each well and incubated for 3 h. A 96-well microtiter plate reader (Molecular Devices, USA) was used to determine the absorbance at $450 \mathrm{~nm}$ for CCK-8. The mean concentration in each set of three wells was measured.

\section{Cell morphology and nuclear fragmentation assay by DAPI} staining

The cells were plated in 24 -well plates at $37^{\circ} \mathrm{C}$ in a humidified atmosphere of $5 \% \mathrm{CO}_{2}$. After $24 \mathrm{~h}$, when the cells had reached $50-60 \%$ confluence, they were treated with various concentrations of APRE. For the cell morphology experiment, the culture plates were examined under a Bright-Field Microscope (20X) and photographed.

SH-SY5Y cells were grown on round coverslips in 24-well plates until the cells reached $80 \%$ confluence. The cells were treated with 0 or $6 \mu \mathrm{g} / \mathrm{ml}$ APRE. After $24 \mathrm{~h}$, the media was removed; the cells were washed with $1 \mathrm{X}$ PBS, and $500 \mu \mathrm{l} 4 \%$ PFA was applied for $15 \mathrm{~min}$. Following this, the cells were washed with PBS and permeabilized with $0.1 \%$-Tween PBS (PBST) for $10 \mathrm{~min}$, and washed again with PBS. The nuclei were then denatured with $2 \mathrm{~N} \mathrm{HCl}(300 \mu \mathrm{l})$ for $10 \mathrm{~min}$ and subsequently washed three times and incubated with $0.1 \mu \mathrm{g} / \mathrm{ml}$ DAPI (1:1000) in PBST for $1 \mathrm{~h}$. After staining, the cells were washed twice with PBS, fluorescent mounting medium was added (Dako North America, USA) and left to dry at room temperature.

\section{Immunocytochemical staining for cleaved caspase-3}

SH-SY5Y cells were grown on round coverslips in 24-well plates until the cells reached $80 \%$ confluence. The cells were treated with 0 or $6 \mu \mathrm{g} / \mathrm{ml}$ APRE. After $24 \mathrm{~h}$, the media was removed, the cells were washed with 1X PBS, and $500 \mu \mathrm{l} \mathrm{3-4 \%}$ PFA was added for $15 \mathrm{~min}$ at room temperature. Subsequently, the cells were washed with PBS and permeabilized with PBS containing $0.25 \%$ Triton $\mathrm{X}-100$ for $10 \mathrm{~min}$. The cells were then washed three times for 5 min with PBS and incubated with $1 \%$ BSA in PBST for 30 min to block non-specific binding of the antibodies. The cells were then incubated with the anticaspase-3 antibody (1:400) in 1\% BSA in PBST overnight at $4^{\circ} \mathrm{C}$. The following day, the cells were washed three times for 5 min with PBS and incubated with the secondary antibody $(1: 1000)$ and DAPI $(0.1 \mu \mathrm{g} / \mathrm{ml})$ in $1 \%$ BSA for $1 \mathrm{~h}$ at room temperature in the dark. Subsequently, the cells were washed three times for 5 min in the dark and then the coverslip was mounted using fluorescent mounting medium (Dako North America) and left to dry at room temperature.

\section{Detection of apoptotic cells by Annexin V assay}

Cells were cultured on 6-well plates and apoptotic cells were measured by Annexin V-FITC/7-AAD staining with the conventional protocol. Then cells were collected and digested into single cell suspensions with EDTA-free trypsin and then subjected to $10 \mu \mathrm{M}$ Annexin V-FITC and $5 \mu \mathrm{M}$ PI solutions added to each cell suspension and incubated for $15 \mathrm{~min}$ at room temperature in dark. The stained cells were calculated by flow cytometry within one hour after staining. Data were analyzed using Cyflogic software (Cyflogic Team, Finland).

\section{Analysis of mitochondrial membrane potential}

Cells were grown on 6 well dishes in $2 \mathrm{ml}$ medium until the cells reached confluence. After incubation $6 \mu \mathrm{g} / \mathrm{ml}$ of APRE for $24 \mathrm{~h}$, the cells were incubated in $10 \mu \mathrm{M}$ of Rhodamine- 123 for $30 \mathrm{~min}$ in dark. The fluorescence intensity of the stained cells was determined by flow cytometry (BD FACSCalibur) (BD Biosciences, USA) using an FL-1H filter. Data were analyzed using Cyflogic software (Cyflogic Team).

\section{Determination of ROS accumulation}

Neuroblastoma cells were grown on 6-well dishes until the cells reached confluence. After treatment with APRE, cells were fixed and incubated in $10 \mu \mathrm{M}$ DCHFDA for $20 \mathrm{~min}$ in dark and dye fluorescence intensity was determined by flow cytometry using an FL-1H filter. Data were analyzed using Cyflogic software.

\section{Determination of autophagic cell death}

Neuroblastoma cells were grown on 6-well dishes in $2 \mathrm{ml}$ medium until the cells reached confluence. After treatment with APRE for $24 \mathrm{~h}$, the cells were incubated with $5 \mu \mathrm{M}$ acridine orange (AO) for $20 \mathrm{~min}$ in the dark and dye fluorescence intensity was determined by flow cytometry using an FL-3H filter. Data were analyzed using Cyflogic software.

\section{Transfection of caspase-3 siRNA}

Caspase- 3 levels were manipulated and reduced with small interfering RNA [caspase-3 siRNA (hours): sc-29237, Santa Cruz Biotechnology]. Cells were transiently transfected with siRNA using Lipofectamine 2000 (Invitrogen, USA) according to 
$\boldsymbol{A}$
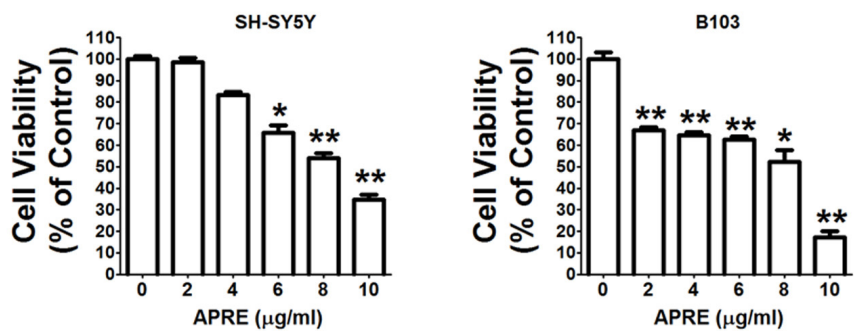

$\boldsymbol{B}$
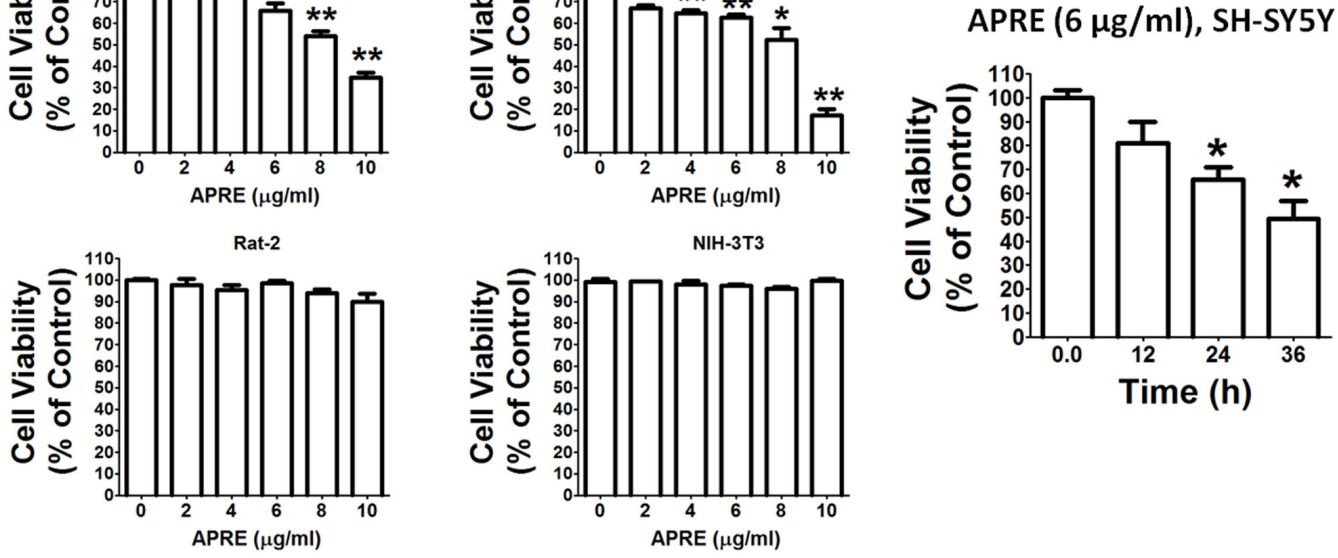

Fig. 1. Anti-proliferative effects of APRE in different cell lines. (A) SH-SY5Y, B103, Rat-2 and NIH 3 T3 cells were cultured in 96-well culture dishes to $50-60 \%$ confluence in DMEM containing $10 \%$ FBS. The cells were treated with various concentrations of APRE. Cell death was determined by using the cytotoxicity assay kit (CCK-8, Dojindo Lab). Each point is mean \pm SE of quintuple of samples. Data is composed of the mean of three independent experiments in which the activity in absence of APRE compared to presence of APRE was significantly different $\left(n=5,{ }^{*} P<0.05,{ }^{* *} P<0.01\right)$. (B) APRE dose of $6 \mu \mathrm{g} / \mathrm{ml}$ was added in a time course experiment. Results are mean \pm SEM and representatives of three independent experiments are shown. Significant differences compared with untreated and APRE added for different times are indicated $\left(n=5,{ }^{*} P<0.05\right)$.

the manufacturer's protocol. Briefly, SH-SY5Y cells were seeded in 6-well culture plates in $2 \mathrm{ml}$ of growth medium without antibiotics. For transfection, Lipofectamine 2000 was lightly mixed before being diluted in DMEM medium without serum and incubated for $5 \mathrm{~min}$ at room temperature. After the incubation, diluted Lipofectamine 2000 was mixed with the diluted siRNA oligomer (resulting concentration of RNA was $40 \mathrm{nM}$ ) in medium, gently mixed and incubated for further $20 \mathrm{~min}$ at room temperature. Next, $100 \mu \mathrm{l}$ of transfection complex was added to each well containing cells and medium and incubated at $37^{\circ} \mathrm{C}$ in humidified $5 \% \mathrm{CO}_{2}$ for $48 \mathrm{~h}$. Afterwards, the medium with transfection complex was replaced with the complete growth medium and treated with APRE for another $24 \mathrm{~h}$. Control siRNA (sc-37007, Santa Cruz Biotechnology) was used as a negative control. Cell viability, Western blotting and caspase-3 activity assay using a specific substrate tested the effect of siRNA silencing after $24 \mathrm{~h}$.

\section{Western blot assay}

SH-SY5Y cells were pretreated with various concentrations of APRE as indicated in each figure legend, and then washed twice with ice-cold PBS. The cells were lysed in lysis buffer $(2 \%$ SDS, $\mathrm{Na}_{3} \mathrm{VO}_{4}$ and protease inhibitor cocktail). After incubation on ice for $10 \mathrm{~min}$, the cells were sonicated for $10 \mathrm{~s}$ at $10 \%$ amplitude, and the lysates were centrifuged at $13,000 \times \mathrm{g}$ for 20 min. Supernatants were collected and the protein concentrations were determined using a Bradford assay (Bio-Rad, USA). Equal amounts of protein were separated by SDS-PAGE $(8 \%$ to $15 \%$ reducing gels), transferred to polyvinylidene difluoride membranes (Millipore, USA), and blocked with 5\% non-fat milk. Membranes were incubated with primary antibody overnight at $4^{\circ} \mathrm{C}$, and then washed in TBST $(10 \mathrm{mM}$ Tris, $140 \mathrm{mM} \mathrm{NaCl}$, $0.1 \%$ Tween-20, $\mathrm{pH} 7.6$ ), incubated with the appropriate sec- ondary antibody, and washed again in TBST. Bands were visualized by enhanced chemiluminescence (ECL) and exposed to X-ray film.

\section{Statistical analysis}

Results were expressed as the mean \pm SEM. Statistical significance was analyzed by one-way ANOVA followed by Dunnett's test or a paired t-test using Prism 4 (GradPad Software, USA). $P<0.05$ was considered significant.

\section{RESULTS}

APRE-treatment reduced the viability of NB cells with an anti-proliferative signal

To check whether APRE exerts antitumor effects, we screened APRE on the cell viability on malignant neuroblastoma tumor cells and normal fibroblast cells. We found that with APRE both the human SH-SY5Y and Rat B103 neuroblastoma cells had reduced viability after $24 \mathrm{~h}$ of treatment (Fig. 1A). On the other hand with APRE, Rat-2 and Mouse embryonic NIH 3T3 fibroblast cells, as models of normal cells, showed no cytotoxic effects after $24 \mathrm{~h}$ of incubation (Fig. 1A). From these results neuroblastoma cancer cells were more sensitive than the normal fibroblast cells towards APRE. With human SH-SY5Y neuroblastoma cells, the $\mathrm{IC}_{50}$ dose of APRE was $7.85 \mu \mathrm{g} / \mathrm{ml}$ after $24 \mathrm{~h}$ of incubation.

To determine the time course effects of APRE on neuroblastoma cells, we treated SH-SY5Y cells with APRE for various times. Here with a fixed dose of $6 \mu \mathrm{g} / \mathrm{ml}$ APRE, the cells were exposed for 0 to $36 \mathrm{~h}$ to determine effect on cell proliferation. We found that as with an increase in incubation time, the percentage of viable cells significantly decreased with respect to the control sets (Fig. 1B). 
A

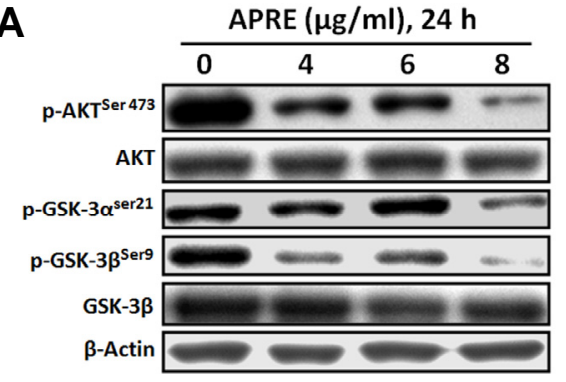

C

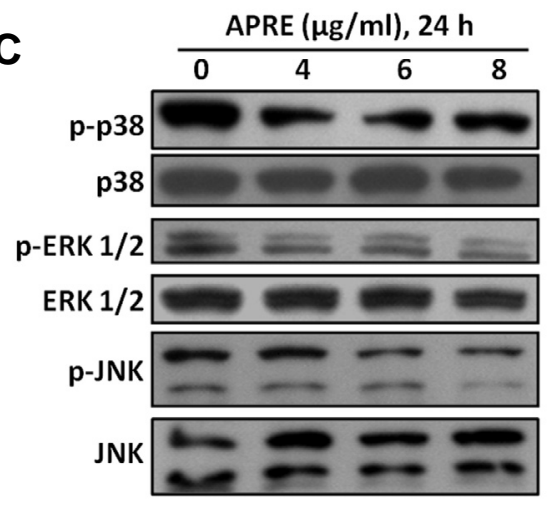

B

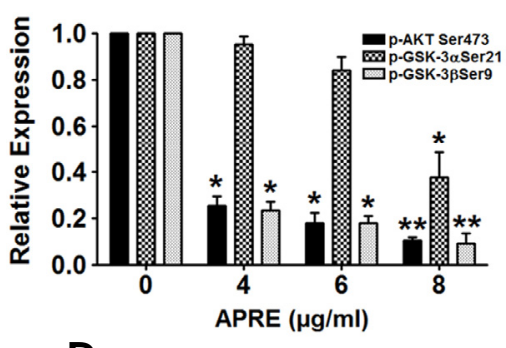

D

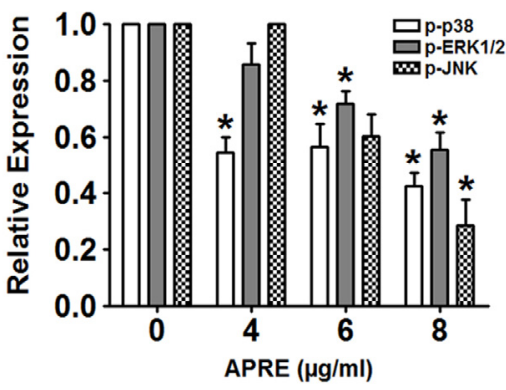

Fig. 2. Effects of APRE on AKT/GSK-3B and MAPK signaling pathway. (A, C) SH-SY5Y cells were cultured and treated with APRE for $24 \mathrm{~h}$. Whole cell lysates were subjected to $10 \%$ SDSPAGE and the levels of p-AKT Ser473, AKT, p-GSK-3 $\alpha$ Ser21, p-GSK-3 3 Ser9, GSK-3 $\beta, \quad p-p 38, \quad$ p38, p-ERK1/2, ERK1/2, p-JNK and JNK proteins were detected by Western blotting. (B, D) The protein band intensities were determined by densitometric scanning and analyzed by Bio-Profil software and the expression levels were normalized to beta-actin. Results are expressed as mean \pm SE and representatives of three independent experiments are shown (n $=3,{ }^{*} \mathrm{P}<0.05,{ }^{* *} \mathrm{P}<0.01$ )

$\boldsymbol{A}$
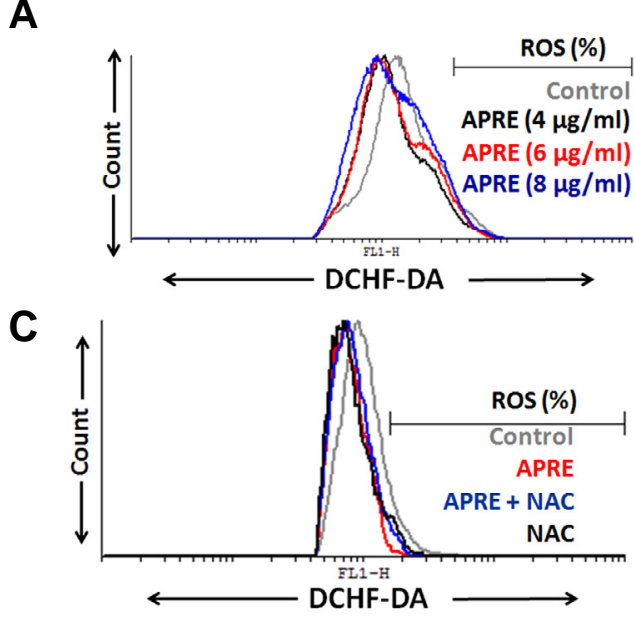

E

APRE $(\mu \mathrm{g} / \mathrm{ml}), 24 \mathrm{~h}$

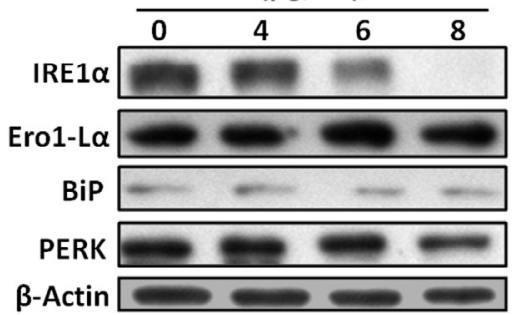

B

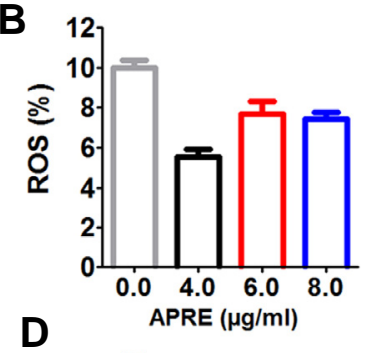

D
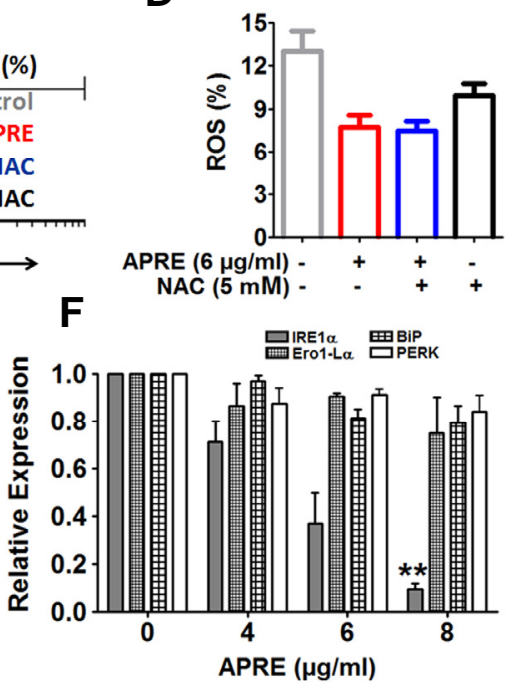

Fig. 3. ROS and ES stress-mediated pathways were not involved in neuroblastoma cell death. (A) ROS production was determined by flow cytometry using DCFHDA. (B) Statistical analysis of ROS production from flow cytometry was done with cyflogic software. "Results" are expressed as mean \pm SE with representatives of three independent reads shown (n $=3$ ). (C) Cells were pretreated with $5 \mathrm{mM}$ NAC for $1 \mathrm{~h}$ followed by in another $24 \mathrm{~h}$ by APRE and identification of ROS using oxidized DCFHDA by flow-cytometry. (D) Statistical analysis of ROS was determined by using cyflogic software. (E) The expression levels of ER stress protein IRE $1 \alpha$, Ero1-L $\alpha, B$ BiP and PERK were detected by Western blotting. (F) The expression of the proteins were analyzed by densitometric scanning. Results are expressed as mean \pm SE and representatives of three independent experiments are shown $\left(n=3,{ }^{* *} P\right.$ $<0.01$ ) 

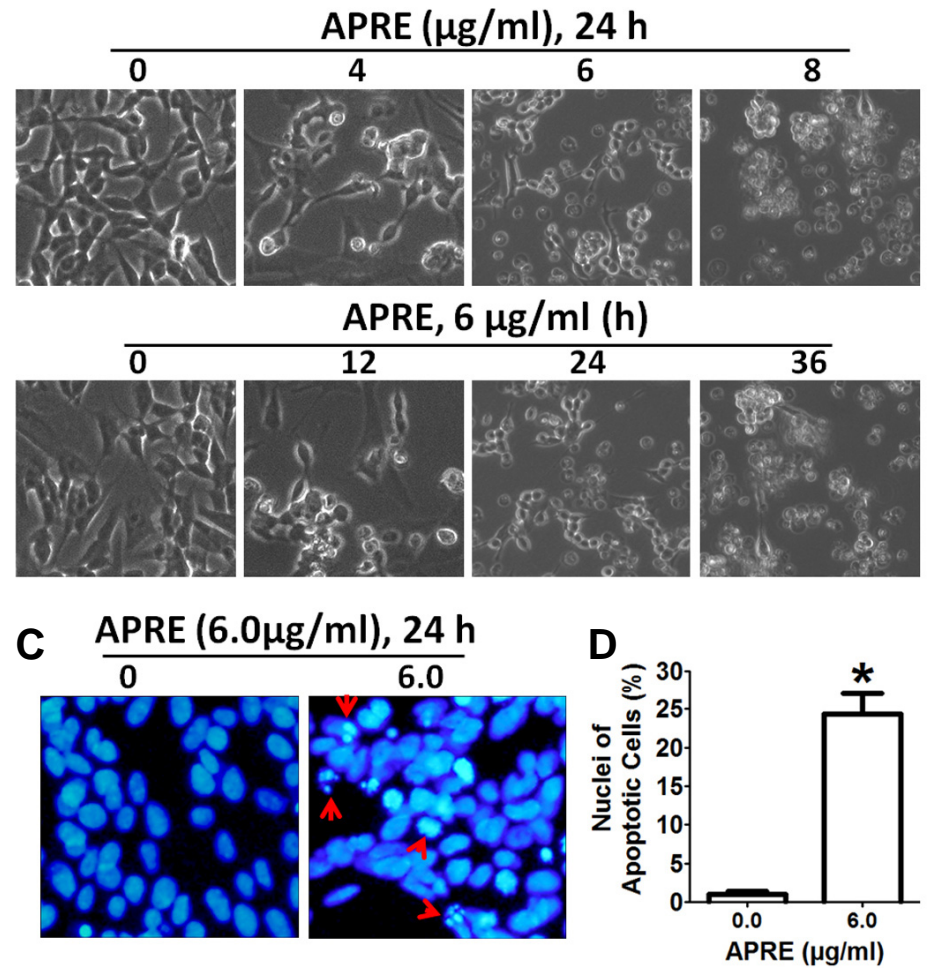

Fig. 4. APRE induced cellular morphology changes in SH-SY5Y typical of apoptosis induction. (A, B) Cells were grown in 24-well culture dishes to $50 \%$ confluence and then treated with 0 to $8 \mu \mathrm{g} / \mathrm{ml}$ APRE for $24 \mathrm{~h}$ for a dose-dependent experiment and with $6 \mu \mathrm{g} / \mathrm{ml}$ APRE for a timedependent experiment and morphology was observed by bright-field microscopy. (C) Apoptotic nuclear morphology was visualized by DAPI staining (arrow indicates fragmented DNA). (D) The percentages of DAPI stained cells were counted in three independent random areas. Results are mean \pm SE and representatives of three independent experiments are shown $(n=$ 3, * $\mathrm{P}<0.05)$. (E, F) Cells were stained with Annexin V-FITC/7-AAD before cell apoptosis analysis by flow cytometry. Results are expressed as mean \pm SE and representatives of three independent experiments $\left(n=3,{ }^{* * *} P<0.001\right)$.

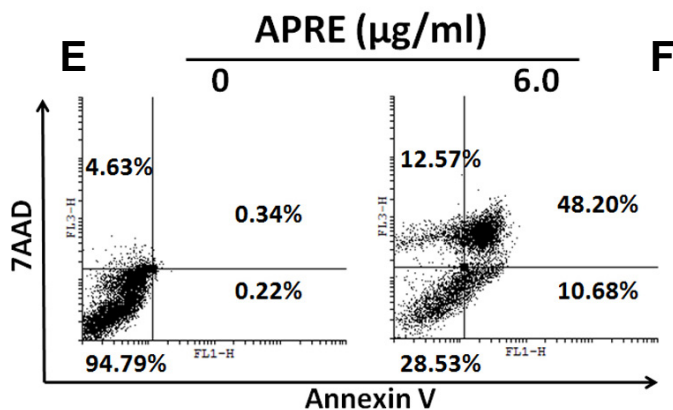

$\boldsymbol{F}$

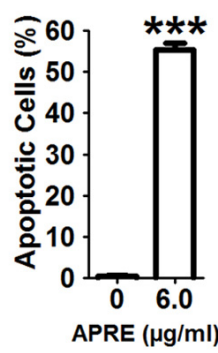

We sought to investigate whether APRE mediates apoptosis of SH-SY5Y cells and whether this is through the activation of caspases. We, therefore, further evaluated the mechanism behind the anti-proliferative effects of APRE on human $\mathrm{SH}$ SY5Y neuroblastoma cells.

\section{APRE inhibited AKT/GSK-3ß and MAPKs but without involving reactive oxygen species and ER stress}

To characterize the role of AKT/GSK-3 and MAPK with APRE on SH-SY5Y cells, we investigated the phosphorylation of AKT, GSK-3, and MAPKs. As shown in Figs. $2 A$ and 2B, phosphorylation of AKT was dose-dependently down regulated; however, APRE had no effect on the level of expression of the total AKT protein. Furthermore with APRE, there was a down-regulation of phosphorylated GSK-3, specifically GSK-3 $\alpha^{\text {Ser21 }}$ and GSK$3 \beta^{\text {Ser9 }}$ (Figs. 2A and 2B), suggesting that APRE inhibited the AKT/GSK-3 $\beta$ the signaling axis. To study whether APRE possesses a multi-kinase inhibitory effect on MAPKs, we tested SH-SY5Y in presence and absence of APRE. We found that with APRE, the phosphorylation levels of p38, ERK1/2, and JNK were reduced significantly in NB cells (Figs. $2 \mathrm{C}$ and $2 \mathrm{D}$ ). Therefore, from these results we could suggest that APRE negatively impacts the proliferative potential of NB cells via deactivation of MAPKs.

To determine any effects of APRE on levels of different stress granules like ROS and thus effecting protein miss-folding signals in endoplasmic reticulum (ER) by producing ER stress, we measured ROS accumulation and ER stress related protein expressions. With APRE treatment, there was a decrease of ROS accumulation (Figs. $3 \mathrm{~A}$ and $3 \mathrm{~B}$ ). We then compared our finding with a known ROS scavenger ( $\mathrm{N}$-acetyl cysteine) and found that APRE may act as an anti-oxidant in the cancer cells and by scavenging the regular ROS elements APRE may mediate its anti-proliferative signals (Figs. $3 C$ and $3 D$ ). Further we compared cellular viability with NAC and APRE and found that NAC had no role on cellular viability, but APRE reduced the viability by $\sim 30 \%$ (viability comparison data not shown).

To examine the effect of APRE on different ER stress markers, we treated SH-SY5Y cells with or without APRE and responses were assayed by immunoblotting for the relevant markers. We found that APRE-treatment did not increase IRE1 $\alpha$, Ero1-L $\alpha, \mathrm{BiP}$ and PERK expression in a dose dependent manner (Figs. 3E and 3F); instead, APRE was able to decrease the expression of IRE1 $\alpha$. So APRE appears to counter ER stress. 
A

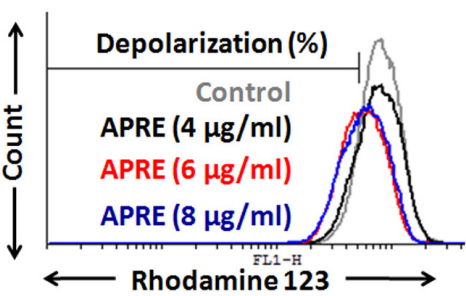

C

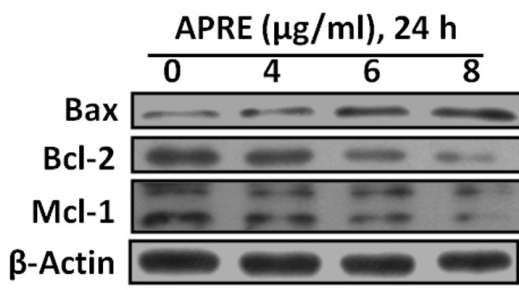

E

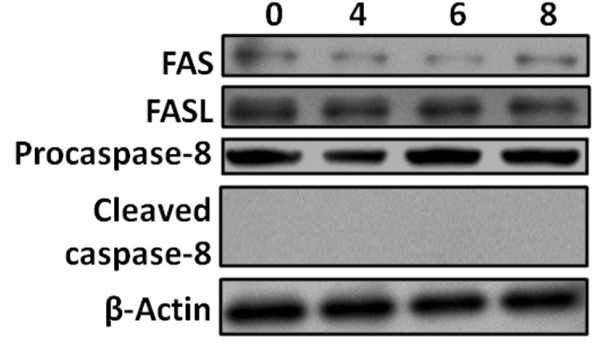

$B$

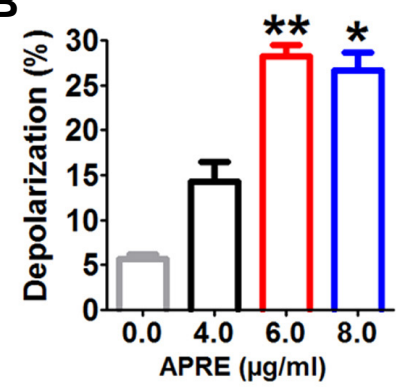

D

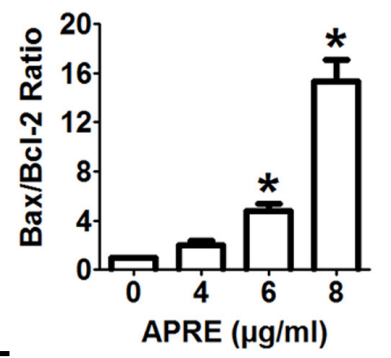

$\boldsymbol{F}$

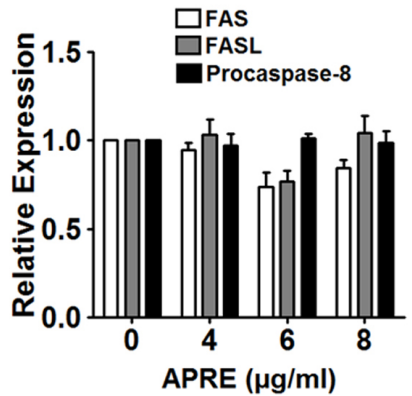

Fig. 5. APRE apoptosis involved intrinsic apoptotic signaling pathways. (A) Depolarization of mitochondrial membrane was measured by flow cytometry using Rhodamine-123 dye. (B) Statistical analysis of depolarization was determined by using cyflogic software. (C) Whole cell lysates were subjected to 15\% SDS-PAGE and the levels of $\mathrm{Bax}$ and $\mathrm{Bcl}-2$ were detected by Western blotting. (D) The intensity of the $\mathrm{Bax}$ and $\mathrm{Bcl}-2$ bands were determined by densitometric scanning and analyzed by Bio-Profil software. Data was composed of the mean \pm SE and representatives of three independent experiments are shown ( $\left.n=3,{ }^{*} P<0.05\right)$. (E) Whole cell lysates were subjected to $10 \%$ SDS-PAGE and the levels of Fas, FasL, and caspase-8 proteins were detected by Western blotting. $(F)$ The expression of the proteins was analyzed by densitometric scanning.
Although we looked at cancer cells, APRE may ameliorate ER stress from aging, genetic mutations, or environmental factors along with indications such as diabetes, inflammation, and neurodegenerative disorders including Alzheimer's disease and Parkinson's disease (Shah et al., 2015). Further study in the context appropriate disease models is required to explore this possibility. Taken together, we found that APRE induced cell death was not associated with ROS and ER stress but with inhibiting different proliferative signals in human SH-SY5Y neuroblastoma cells.

\section{APRE-induced cell death was due to an apoptotic signal}

To observe the morphological changes with APRE, we incubated SH-SY5Y cells in a dose- and-time dependent manner and digitally imaged under a bright field microscope. We found that APRE, cells were rounded with neurite retraction and membrane blebbing (Figs. 4A and 4B). Also chromatin condensation and DNA fragmentation were observed by DAPI staining and both are known indicators of apoptosis in the cell lines (Fig. 4C). The cellular and nuclear morphological changes cumulatively indicated APRE-treatment may result in apoptosis (Fig. 4D).

Up next after obtaining morphological changes with APRE, we stained the cells with Annexin $V$ and 7-AAD to check whether the morphological changes were due to induction of apoptosis. We found that the apoptotic cells population (Annexin $\mathrm{V}$ positive/7-AAD negative and Annexin $\mathrm{V}$ positive/7-AAD positive) was significantly increased with APRE treatment (Figs $4 \mathrm{E}$ and $4 \mathrm{~F}$ ). Taken together, we can suggest that the morphological changes of NB cells by APRE were from apoptosis induction.

\section{APRE-induced apoptosis was from an intrinsic caspase- dependent signal rather than from extrinsic signals}

To examine whether APRE treatment involves mitochondria during the apoptosis process, we investigated depolarization of mitochondrial membrane (MMP) by flow cytometry analysis using rhodamine-123 staining. Results indicated that APRE was able to depolarized mitochondrial membrane potential in SH-SY5Y cells (Figs. 5A and 5B).

To understand the molecular mechanisms involved in the activation of cell death induced by APRE, we evaluated the modulation of expression levels of the anti-apoptotic and proapoptotic Bcl-2 family of proteins in SH-SY5Y cells. The results indicated that the expression of Bax in SH-SY5Y cells was significantly up-regulated with APRE and in a dose-dependent manner. Furthermore, the expression levels of anti-apoptotic proteins $\mathrm{Bcl}-2$ and $\mathrm{Mcl}-1$ were reduced in a dose-dependent manner ( 0 to $8 \mu \mathrm{g} / \mathrm{ml}$ for $24 \mathrm{~h}$ ) as compared to the control (Fig. $5 \mathrm{C})$. By densitometry analysis, the level of the Bax and Bcl-2 ratios were significantly increased with APRE-treatment (Fig. 5D). This observation suggests that APRE-treatment can alter the protein expression levels of key members of the Bcl-2 family, which may contribute to the susceptibility of cancer cells in 


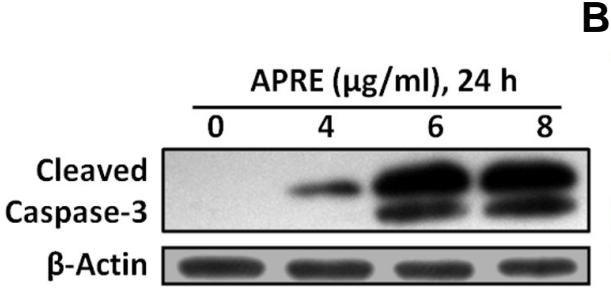

C

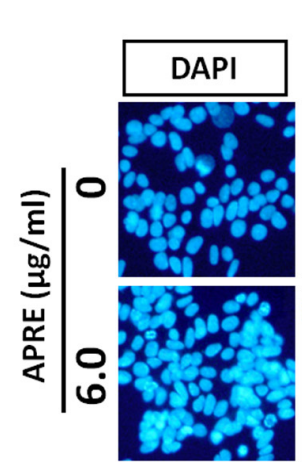

$B$

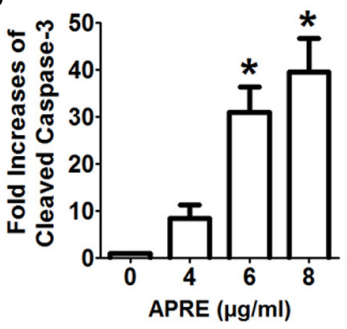

$D$

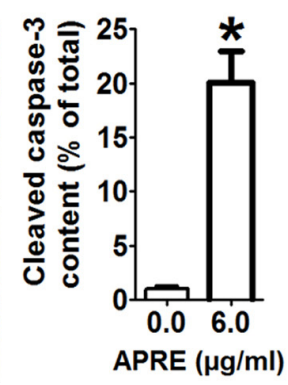

$E$
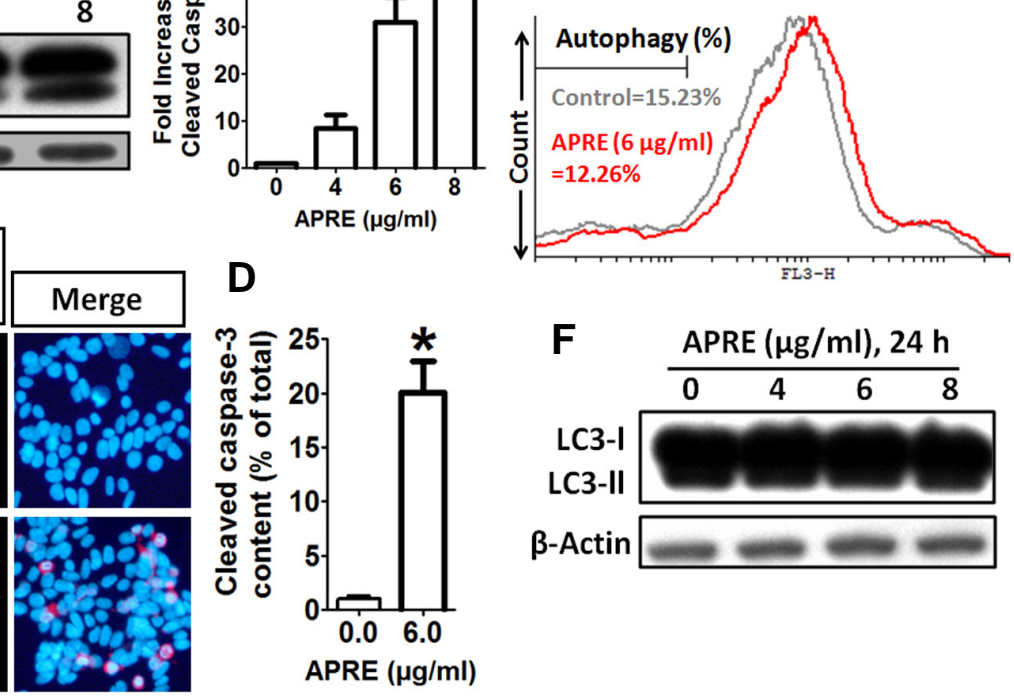

$\boldsymbol{F}$

$\operatorname{APRE}(\mu \mathrm{g} / \mathrm{ml}), 24 \mathrm{~h}$

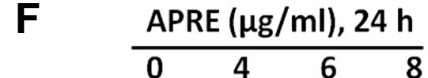

LC3-I

LC3-II

$\beta$-Actin

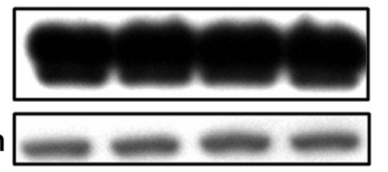

Fig. 6. APRE altered the expression of apoptosis-related protein caspase-3. (A) Whole cell lysates were subjected to 15\% SDS-PAGE and the levels of caspase- 3 proteins were detected by Western blotting. (B) The intensity of the cleaved caspase-3 bands were determined by densitometric scanning and analyzed by Bio-Profil software. Data was composed of the mean \pm SE and representatives of three independent experiments are shown $\left(n=3,{ }^{*} P<0.05\right)$. (C) Red fluorescence (Alexa Fluor 568 goat anti-rabbit) indicates cleaved caspase-3 expression, whereas the nucleus is stained blue (DAPI). (D) The percentages of cleaved caspase-3 contents cells were counted in three independent random areas $\left(n=3,{ }^{*} p<0.05\right)$. (E) Cells were exposed to 0 and $6 \mu \mathrm{g} / \mathrm{ml}$ APRE for $24 \mathrm{~h}$ and autophagy was analyzed by flow cytometry following acridine orange (AO) staining. (F) Whole cell lysates were subjected to $15 \%$ SDS-PAGE and the levels of LC3 was detected by Western blotting.

mitochondrial dysfunction and initiates the intrinsic pathway of apoptosis.

Further to check whether the extrinsic apoptotic pathway was also involved during the cytotoxicity by APRE, we examined changes in the levels of Fas receptor, Fas ligand and caspase8 by Western blotting. We observed no significant changes in the expression of the Fas receptor or Fas ligand and cleaved caspase-8 in SH-SY5Y cells after incubation with APRE for 24 $h$ (Figs. 5E and 5F). Together, these findings indicate that APRE can initiate mitochondria dysfunction to induce apoptosis but APRE does not involve extrinsic apoptosis signals.

To further determine the details of APRE treatment on the caspase pathway, we incubated SH-SY5Y cells in the absence or presence of APRE and then harvested the cells for Western blotting analysis. Incubation of SH-SY5Y cells with APRE upregulated the expression of active cleaved caspase-3, thereby activating the apoptotic cascade pathway (Fig. 6A). As shown by densitometry, APRE significantly up-regulated cleaved caspase-3 expressions in SH-SY5Y cells as compared to the control cells (Fig. 6B), indicating that APRE-induced apoptosis is mediated by the activation of an intrinsic caspase pathway. Moreover, immunofluorescence analysis revealed that APRE (6 $\mu \mathrm{g} / \mathrm{ml}, 24 \mathrm{~h}$ ) significantly enhanced cleaved caspase-3 expression within the nucleus compared with the control (Figs. 6C and 6D).

Further we tried to evaluate the role of non-caspase death pathway, the autophagy-mediated cell death. We stained the cells with $\mathrm{AO}$ and estimated acidic vacuoles formation with flow cytometry using the FL-3H filter and along with that we studied autophagy marker protein LC3 expression by Western blot. We found that with APRE there was no autophagy mediated cell death (Figs. 6E and 6F), indicating that apoptosis was the sole cell-death pathway activated with APRE.

We inhibited caspase activity by using a pan caspase inhibitor, DEVD-fmk and by silencing caspase-3 expression by targeting caspase-3 with siRNA, and determined their effect on cell viability assay and protein expression in presence of APRE. Here we found that as expected APRE induced cellular cytotoxicity, but in presence of DEVD-fmk, the efficacy of APRE on the NB in inducing cell death greatly diminished as compared to the only APRE treated cells. (Fig. 7A). Immunoblotting confirmed that treatment of cells with APRE significantly increased the levels of cleaved caspase-3, and co-treatment with APRE and caspase-3 inhibitor significantly down-regulated cleaved caspase-3 expression (Figs. 7B and 7C). Moreover, we found that in presence of DEVD-fmk, APRE could not bring about the apoptotic morphological changes in SH-SY5Y cells, as seen with APRE alone treatment (Fig. 7D). Also, cell viability was significantly increased with APRE when cells were transfected with caspase-3 siRNAs as compared to control siRNA treated SH-SY5Y cells (Fig. 7E). Protein expression study confirmed that siRNA transfected cells had significantly reduced levels of caspase-3 (Fig. 7F).

Taken together, in presence of APRE, caspase- 3 is activated, mediating apoptosis in the NB cells. In addition, APRE also could depolarize mitochondria membrane potential without involving the extrinsic death pathway and also without involving non-apoptotic pathway like autophagy. 
$\boldsymbol{A}$

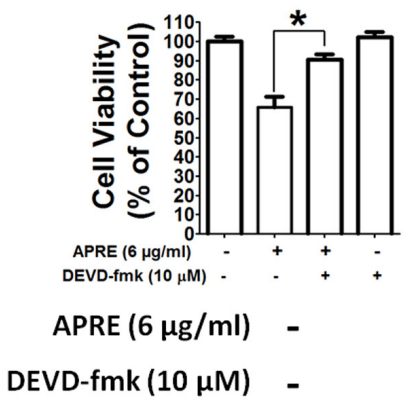

D

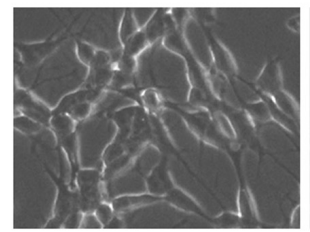

$E$

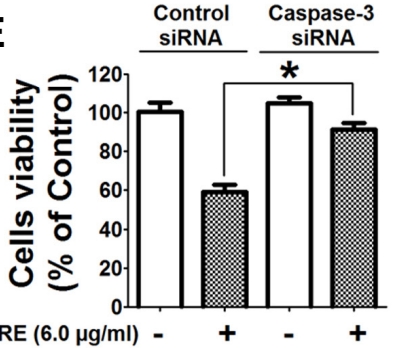

$B$

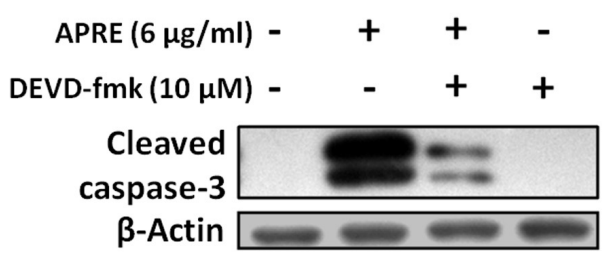

C

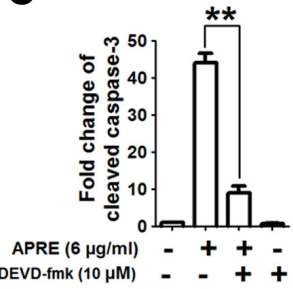

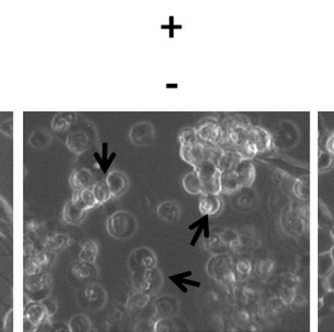
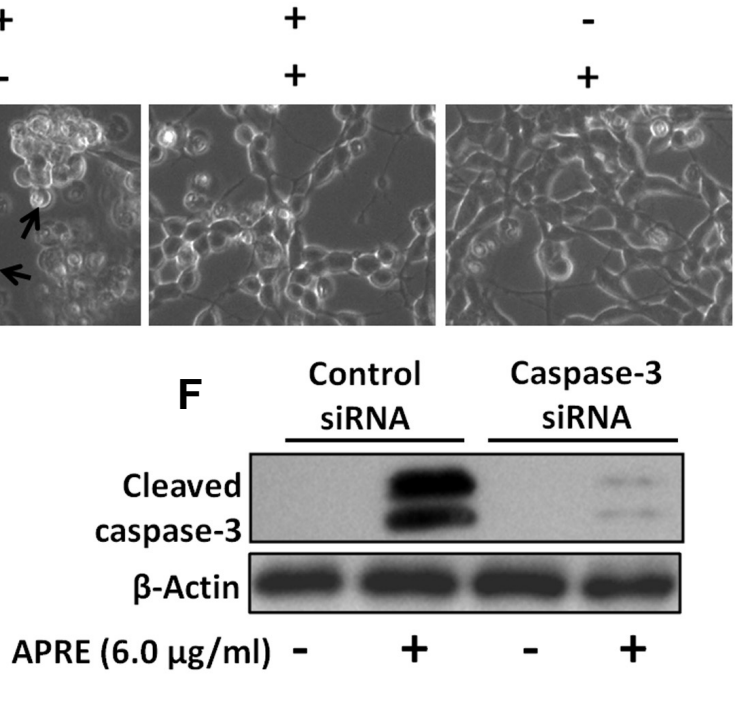

Fig. 7. APRE-induced apoptosis in SH-SY5Y cells was caspase-3 dependent. (A) Cells were pretreated with $10 \mu \mathrm{M}$ DEVD-FMK for $1 \mathrm{~h}$ just before exposure to $6 \mu \mathrm{g} / \mathrm{ml}$ of APRE for $24 \mathrm{~h}$ and viable cells' number was determined by using the cytotoxicity assay kit. Results are means \pm SE and representatives of three independent experiments are shown $\left(n=3,{ }^{*} P<0.05\right)$. (B) Whole cell lysates were subjected to $15 \%$ SDSPAGE and the levels of cleaved caspase- 3 were detected by Western blotting. (C) The band intensity of cleaved caspase- 3 was determined by densitometric scanning and analyzed by Bio-Profil software. Data was composed of the mean $\pm \mathrm{SE}$ and representatives of three independent experiments are shown $\left(n=3,{ }^{* *} P<0.01\right)$. (D) Cells were grown in 24-well culture dishes to near confluence $50 \%$ and after treatment, morphology was observed by Bright-Field Microscopy. (E) SH-SY5Y cells were transfected with control and caspase-3 siRNA for $48 \mathrm{~h}$ followed by treatment with $6 \mu \mathrm{g} / \mathrm{ml}$ of APRE for $24 \mathrm{~h}$. Cell number was quantified by CCK-8 kit. Results are expressed as mean \pm SE and representatives of five independent experiments $\left(n=5,{ }^{*} P<0.05\right)$. (E) The levels of cleaved caspase- 3 were detected by Western blotting using $15 \%$ SDS-PAGE.

\section{DISCUSSION}

The present study was designed to define the mechanism(s) of the anti-proliferative and cell death inducing potential of natural plant extracts from our screening effort on NB cells; this was to further the efforts for potential novel approaches in management of neuroblastoma. Our study emphasizes that neuroblastoma cancer cells show relatively higher toxicity than normal fibroblast cells (Fig. 1), suggesting that APRE may be a specific anticancer agent. To date, to our knowledge, there has been no study describing the anticancer effects of APRE. The purpose of the present study was to investigate whether APRE can induce apoptosis of SH-SY5Y cells, explaining the mechanisms underlying either the anti-proliferative or cytotoxic nature of APRE against NB cells.

The results presented herein suggest that instead of the Fas/FasL signaling pathway, APRE down-regulates members of the Bcl-2 family of anti-apoptotic proteins, and up-regulates pro-apoptotic proteins. The down-regulation of the PI3K/AKT/GSK-3 $\beta$ pathway along with MAPKs could be the pathway for the anti-proliferative for APRE and in addition, lead to induction of mitochondria-caspase pathway resulting in DNA fragmentation and apoptosis (Figs. 2 and 4). Reduction in cell viability with APRE in SH-SY5Y cells by APRE may provide us an important lead in therapeutic efforts in neuroblastoma. APRE showed more of an effect towards NB cancer cells than the models of normal cells like NIH3T3 fibroblast cells. This dichotomy in the sensitivity to APRE may be due presence of particular targets in cancer cells not present in untransformed cells such as proteins with activating mutations (Flaherty et al., 2012). Here, we found that APRE targeted the growth related MAPKs and Akt to induces cytotoxicity in the cancer cells.

Apoptosis, as a regulated mode of cell death, includes two major pathways, the death-receptor-mediated extrinsic pathway and the mitochondria-dependent intrinsic pathway (Castel et al., 2007; Maris et al., 2007). Bcl-2 family proteins play important roles in controlling the mitochondria-dependent intrinsic pathway (Kroemer, 1997). To date, more than 20 members of the $\mathrm{Bcl}-2$ family have been identified including anti-apoptotic proteins (such as Bcl-2, Bcl-xL) and pro-apoptotic proteins (such 
as Bax, Bak) (Besbes et al., 2015). And with many tumor types there is overexpression of anti-apoptotic proteins, particularly Bcl-2 (Reed, 1997). Bcl-2 inhibits apoptosis as well as its close homologues - Bcl-XL, Bcl-W, Mcl-1 and a1. All these share four $\mathrm{Bcl}-2$ homology $(\mathrm{BH})$ domains. Other relatives that are similar in sequence and structure - BAX, BAK- instead promote apoptosis (Nascimento et al., 2004).

Exposure to different stresses pharmacologically or via ROS results in activation of $\mathrm{BH} 3$-only proteins, which deactivate the pro-survival signals and subsequently activate Bax and Bak leading to mitochondrial membrane permeabilization (Besbes et al., 2015; Yee et al., 2009). Anti-cancer effects of many currently available chemotherapeutic agents may be inhibited by the up-regulation of $\mathrm{Bcl}-2$ expression to block the apoptotic pathway (Bodur and Basaga, 2012). Thus, antagonizing the function of $\mathrm{Bcl}-2$ may be a useful strategy for sensitizing cancer cells to apoptosis induction such as chemotherapy (Kwon et al., 2015). Conversely Bax as a pro-apoptotic member of the Bcl-2 family was shown to constitute a requisite gateway to the mitochondria-dependent pathway of apoptosis (Estaquier et al., 2012). Thus, restoring the sensitivity of cancer cells to antitumor agents can also be carried out by up-regulating Bax expression (Zhang et al., 2004). The balance between the expression levels of Bcl-2 and Bax is critical in determining the fate of cells with respect to survival or death. In the present study, APRE dose-dependently down-regulated the level of Bcl2 and up-regulated the level of Bax (Fig. 5). This may indicate that APRE-induced apoptosis strongly correlates with the intrinsic mitochondrial-dependent apoptotic signaling pathway. Further details on the pathways and mechanisms of APRE induced apoptosis in SH-SY5Y neuroblastoma cells warrant additional research.

In cancer biology, it is now evident that many cancer cells circumvent normal apoptotic mechanisms to prevent their selfdestruction. The caspases are key executioners of apoptosis, triggered via endoplasmic reticulum stress, extracellular stimuli or mitochondrial damage (Fiandalo and Kyprianou, 2012). In particular, caspase- 3 plays a pivotal role in the terminal and execution phases of apoptosis induced by diverse stimuli (Hector and Prehn, 2009). Upon activation, initiator caspase-9 triggers the proteolytic activation of the executioner caspase-3/7 and caspase- 8 in a process that results in the cleavage of PARP and subsequent DNA degradation and apoptotic death (Hoye et al., 2008). In the present study, treatment of SH-SY5Y cells with APRE resulted in a dramatic increase in the proteolytic activation of caspase- 3 , which is the main executioner of apoptosis. This study examined whether caspase- 3 , which is a substrate of caspase-9, is cleaved in cells exposed to APRE (Fig. 6). As expected, caspase-3 was clearly degraded in a concentration-dependent manner, which correlated with a caspase signaling pathway and apoptosis. Under the same experimental conditions, z-DEVD-fmk and caspase-3 siRNA prevented APRE-induced apoptosis by blocking caspase activation (Fig. 7). Therefore, APRE may be used as a potential apoptosis inducing agent in neuroblastoma cancer cells for the development of anticancer drugs.

In the present study, we found that APRE down-regulated the phosphorylation of AKT, which is closely linked to cell survival and proliferation (Fig. 2). One of the direct downstream targets

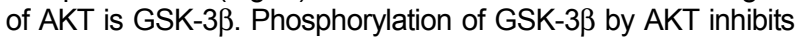
its own enzymatic activity as a serine/threonine kinase (Roy and Nicholson, 2000). As a consequence of the activation of PI3K signaling, GSK-3 $\beta$ activity is attenuated by activated AKT. When active, GSK-3 $\beta$ can phosphorylate a number of protein

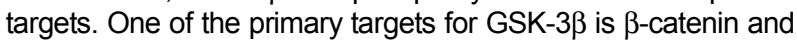

which is phosphorylated and further degraded by proteasomes (Takahashi-Yanaga and Sasaguri, 2009). Inhibition of Akt-GSK signaling by APRE may mediate the decreases in Bcl-2 and increases in Bax protein levels and thus promote apoptosis in the NB cells. As APRE treatment was not able to increase FAS protein and caspase 8 expression, APRE probably has no role in an extrinsic death pathway in NB cells. From these observations, we suggest that the reduction in proliferation and tumor cell viability along with induction of apoptosis by APRE could be the result of PI3K/AKT/GSK-3 $\beta$ signaling inhibition.

In conclusion, in the present study we observed apoptosis induction as the mechanism underlying APRE-induced cellular cytotoxicity seen in human neuroblastoma cells but not in fibroblast cells as models of normal cells. As far as we know, this is the first report to demonstrate that APRE dose-dependently induces apoptosis in neuroblastoma cells by activating the caspase signaling as triggered by changes in the level of Bcl-2 family proteins, culminating in fragmentation of DNA along with other apoptosis phenotypes in SH-SY5Y cells. These findings suggest that APRE may serve as a potent chemo-sensitizer in the treatment of human cancers by modulating the levels of relevant $\mathrm{Bcl}-2$ family proteins. Our results warrant further investigation of APRE as a candidate agent in neuroblastoma treatment possibly exploring efficacy in in vivo experimental neuroblastoma models.

\section{ACKNOWLEDGMENTS}

This research was supported by Research Grant through the National Research Foundation of Korea (NRF) funded by the Ministry of Education (NRF-2015R1A2A2A01007473, 2013M3C7A1056568) and by Hallym University (HRF-G-20153 ), South Korea. This manuscript was edited by Dr. Alistare Sadra.

\section{REFERENCES}

Besbes, S., Massoud, M., Marc, Pocard., and Christian, B. (2015). New dimension in therapeutic targeting of BCL-2 family proteins. Oncotarget 6, 12862-12871.

Bodur, C., and Basaga, H. (2012). Bcl-2 inhibitors: emerging drugs in cancer therapy. Curr Med Chem. 19, 1804-1820.

Brodeur, G.M. (2003). Neuroblastoma: biological insights into a clinical enigma. Nat. Rev. Cancer 3, 203-216.

Castel, V., Grau, E., Noguera, R., and Martinez, F. (2007). Molecular biology of neuroblastoma. Clin. Transl. Oncol. 9, 478483.

Estaquier, J., Francois, V., Jean-Luc V., and Bernard, M. (2012) The mitochondrial pathways of apoptosis. Adv. Exp. Med. Biol. 942,157-83.

Fiandalo, M.V., and Kyprianou, N. (2012). Caspase control: protagonists of cancer cell apoptosis. Exp. Oncol. 34, 165-175

Flaherty, K.T., Infante, J.R., Daud, A., Gonzalez, R., Kefford, R.F., Sosman, J., Hamid, O., Schuchter, L., Cebon, J., Ibrahim, N., et al. (2012). Combined BRAF and MEK inhibition in melanoma with BRAF V600 mutations. N Engl. J. Med. 367, 1694-1703.

Hector, S., and Jochen, H.M P. (2009). Apoptosis signaling proteins as prognostic biomarkers in colorectal cancer: a review. Biochim. Biophys. Acta 1795, 117-129.

Hoye, A.T., Jennifer, E.D., Peter, W., Mitchell, P.F., and Valerian, E.K (2008). Targeting mitochondria. Acc. Chem. Res. 41, 87-97.

Kwon, Y.H., Bishayee, K., Rahman, A., Hong, J.S., Lim, S.S., and Huh, S.O. (2015). Morus alba accumulates reactive oxygen species to initiate apoptosis via FOXO-caspase 3-dependent pathway in neuroblastoma cells. Mol. Cells 38, 630-637.

Kroemer, G. (1997). The proto-oncogene Bcl-2 and Its role in regulating apoptosis. Nat. Med. 3, 614-620.

Maris, J.M., Michael, D.H., Rochelle, B., and Susan, L.C. (2007). Neuroblastoma. Lancet 369, 2106-2120.

Maris, J.M., Mosse, Y.P., Bradfield, J.P., Hou, C., Monni, S., Scott, R.H., Asgharzadeh, S., Attiyeh, E.F., Diskin, S.J., Laudenslager, 
M., et al. (2008). Chromosome 6p22 locus associated with clinically aggressive neuroblastoma. N Engl. J. Med. 358, 25852593.

Nascimento, Pde S., Ornellas, A. A., Campos M. M., Scheiner, M.A. Fiedler, W., and Alves, G. (2004). Bax and bcl-2 imbalance and HPB infection in penile tumors and adjacent tissues. Prog Urol. 14, 353-359.

Pinto, N.R., Applebaum M.A., Volchenboum, S.L., Matthay, K.K., London, W.B., Ambros, P.F., Nakagawara , A., Berthold, F., Schleiermacher, G., Park, J.R., et al. (2015). Advances in risk classification and treatment strategies for neuroblastoma. J. Clin. Oncol. 20, 3008-3017.

Rahman, Md A., Nam-Ho, Kim., and Sung-Oh, Huh. (2013). Cytotoxic effect of gambogic acid on SH-SY5Y neuroblastoma cells is mediated by intrinsic caspase-dependent signaling pathway. Mol. Cell Biochem. 377, 187-196.

Reed, J.C. (1997). Double identity for proteins of the Bcl-2 family. Nature 387, 773-776.

Roy, S., and Nicholson D. W. (2000). Cross-talk in cell death signaling. J. Exp. Med. 192, 21-26.

Schrey, D., Vaidya, S.J., Levine, D., Pearson, A.D., and Moreno, L. (2015). Additional therapies to improve metastatic response to induction therapy in children with high-risk neuroblastoma. J. Pediatr. Hematol. Oncol. 37, e150-e153.

Shah, S.Z., Zhao, D., Khan, S.H., and Yang, L. (2015). Unfolded protein response pathways in neurodegenerative diseases. J. Mol. Neurosci. doi:10.1007/s12031-015-0633-3.

Shi, X., Chen, X., Li, X., Lan, X., Zhao, C., Liu, S., Huang, H., Liu,
N., Liao, S., Song, W., et al. (2014). Gambogic acid induces apoptosis in imatinib-resistant chronic myeloid leukemia cells via inducing proteasome inhibition and caspase-dependent Bcr-Abl downregulation. Clin. Cancer Res. 20, 151-163.

Sonawane, P., Cho, H.E., Tagde, A., Verlekar, D., Yu, A.L., Reynolds, C.P., and Kang, M.H. (2014). Metabolic characteristics of 13-cis-retinoic acid (isotretinoin) and antitumour activity of the 13-cis-retinoic acid metabolite 4-oxo-13cis-retinoic acid in neuroblastoma. Br. J. Pharmacol. 171, 53305344.

Takahashi-Yanaga, F., and Sasaguri, T. (2009). Drug development targeting the glycogen synthase kinase-3beta (GSK-3beta)mediated signal transduction pathway: inhibitors of the Wnt/beta-catenin signaling pathway as novel anticancer drugs. J. Pharmacol. Sci. 109, 179-183.

Wang, J., Zhu, L., Zou, K., Cheng, F., Dan, F., Guo, Z., Cai, Z., and Yang, J. (2009). The anti-ulcer activities of bisabolangelone from Angelica polymorpha. J. Ethnopharmacol. 123, 343-346.

Yang, Y., Zhang, Y., Ren, F.X., Yu, N.J., Xu, R., and Zhao, Y.M. (2013). Chemical constituents from the roots of Angelica polymorpha Maxim. Yao Xue Xue Bao 48, 718-722.

Yee, K.S., Wilkinson, S., James, J., Ryan, K.M., and Vousden, K.H. (2009). PUMA- and bax-induced autophagy contributes to apoptosis. Cell Death Differ. 16, 1135-1145.

Zhang, C.L., Wu, L.J., Tashiro, S., Onodera, S., and Ikejima, T. (2004). Oridonin induces apoptosis of HeLa cells via altering expression of Bcl-2/Bax and activating caspase-3/ICAD pathway. Acta Pharmacol. Sin. 25, 691-698. 